\title{
Winning But Falling Short? Erneuter Sieg der Demokratischen Partei bei den Wahlen zum US-Kongress vom 4. November 2008
}

\author{
Michael Kolkmann
}

\begin{abstract}
Die Wahl von Barack Obama zum 44. Präsidenten der USA am 4. November 2008 fand weltweite Beachtung. Deutlich im Schatten dieses Siegs und auch schon des Wahlkampfes zwischen dem Demokraten Obama und seinem Republikanischen Herausforderer John McCain standen die Wahlen zum 111. US-Kongress. Gleichwohl dürfte auch die Zusammensetzung und Schwerpunktsetzung von Senat und Repräsentantenhaus ${ }^{1}$ in den nächsten Jahren mit darüber entscheiden, ob Obama im präsidentiellen Regierungssystem der USA seine im Wahlkampf formulierte Agenda umsetzen und politisch erfolgreich sein kann. Daher liegt es nahe, das Ergebnis der Kongresswahlen ausführlich zu analysieren und zu fragen, auf welche politischen Verhältnisse im Kongress sich der neue Präsident, aber auch die übrigen Akteure im politischen System der USA, zunächst bis zur nächsten Kongresswahl am 2. November 2010 einstellen müssen.
\end{abstract}

\section{Die Ergebnisse der Kongresswahlen}

Nach einem Jahrzehnt unter Republikanischer Kontrolle (abgesehen vom Zeitraum Mai 2001 bis Januar 2003 im Senat) hatten die Demokraten in den Kongresswahlen vom 7. November 2006 die Mehrheit in beiden Kammern zurückerobert ${ }^{2}$, und schon zu Beginn des Wahljahres 2008 sah die Ausgangslage für sie positiv aus: Vermehrte Rücktritte von Republikanern in Repräsentantenhaus und Senat sowie die zunehmende beziehungsweise lang anhaltende Unpopularität Präsident George W. Bushs sahen die Republikanischen Kandidaten im Nachteil. ${ }^{3}$ Im April 2008 erreichte Bush die niedrigsten Zustimmungsraten, die

1 Vgl. zu den grundsätzlichen Funktionen und elementaren Unterschieden zwischen Repräsentantenhaus und Senat Michael Kolkmann, Handelspolitik im US-Kongress. Die Normalisierung der US-chinesischen Handelsbeziehungen, Opladen 2005, S. 142 - 149.

2 Für einen Rückblick auf die Dekade Republikanischer Mehrheiten im Kongress vgl. Lawrence C. Dodd / Bruce I. Oppenheimer, A Decade of Republican Control: The House of Representatives, 1995 - 2005, in: dies. (Hrsg.), Congress Reconsidered, Washington 2005, S. 23 - 54, und Barbara Sinclair, The New World of U.S. Senators, in: Lawrence C. Dodd / Bruce I. Oppenheimer (Hrsg.), a.a.O., S. 1 - 22; vgl. weiterhin Stuart Rothenberg, The Fight for the Senate, in: Larry Sabato (Hrsg.), The Sixth Year Itch. The Rise and Fall of the George W. Bush Presidency, New York u.a. 2008, S. 65 - 82; David Wasserman, The 2006 House Midterm Maelstrom: From „The GOP’s to Lose“ to a „House of Blues“, in: Larry Sabato (Hrsg.), a.a.O., S. 97 - 133.

3 In acht Zwischenwahlen (den so genannten Special Elections) im Verlaufe des Jahres 2008 konnten die Demokraten bereits Sitze hinzugewinnen: So konnten sie vier eigene Sitze verteidigen und den Republikanern drei Sitze abnehmen, einen Sitz konnten die Republikaner verteidigen; bei zwei Zwischenwahlen im Jahre 2009 konnte am 7. April 2009 im 5. Distrikt von Illinois der Demokrat Mike Quigley den zuvor vom jetzigen Stabschef im Weißen Haus, Rahm Emanuel, gehaltenen Sitz gewinnen, vgl. Deanna Bellandi, Democrat Wins Rahm's Ill. House Seat, in: The Washington Post vom 8. April 2009; in einer weiteren Zwischenwahl am 31. März 2009 im 20. Distrikt von New York konnte sich der Demokrat Scott Murphy gegen den Republikaner Jim 
ein Präsident in den 70 Jahren der Gallup Polls je erzielt hat. 69 Prozent der Befragten waren mit seiner Amtsführung unzufrieden, lediglich 28 Prozent äußerten sich positiv. Im September 2008 variierte die Zustimmung je nach Meinungsforschungsinstitut zwischen 19 und 34 Prozent. ${ }^{4}$

In diesen Umfragewerten spiegelten sich diverse Verfehlungen der Bush-Administration, so etwa der andauernde Krieg im Irak, die umstrittenen Entlassungen von mehreren Staatsanwälten, die laut Justizministerium nicht aggressiv genug gegen Politiker der Demokratischen Partei ermittelten (weshalb Justizminister Alberto Gonzalez gehen musste), das Bekanntwerden eines weit reichenden Überwachungsprogrammes sowie der Folterskandal in Abu Ghraib und die Diskussion um umstrittene Verhörtechniken wie das Waterboarden, zuvor bereits Bushs missratene Reaktion auf den Hurrikan Katrina und die politische Niederlage seines Planes, das Sozialversicherungssystem teilweise zu privatisieren. ${ }^{5}$ Hinzu kamen die weit reichenden Konsequenzen der Hypotheken-, Wirtschafts- und Finanzkrise, die die Schlussphase des Wahlkampfes dominierten und den Kongress zu einer ganzen Reihe von parlamentarischen Aktivitäten veranlassten. ${ }^{6}$ Auf der anderen Seite sind die „Presidential Coattails“ Obamas zu berücksichtigen, also die Wirkung des (erfolgreichen) Präsidentschaftskandidaten auf den Ausgang der Kongresswahlen. Koppelt man das Ergebnis für den Kongress mit dem der Präsidentschaftswahl, fällt auf, dass Obama etwas geschafft hat, was den letzten drei Präsidenten der Demokratischen Partei, die einen Republikanischen Amtsvorgänger ablösten, nicht gelungen war, nämlich Sitze im Kongress hinzuzugewinnen. Bei der Wahl John F. Kennedys verloren die Demokraten 22 Mandate im Repräsentantenhaus und zwei im Senat, unter Jimmy Carter blieb das Verhältnis zwischen den Parteien im Kongress annähernd gleich, und bei Bill Clintons Sieg 1992 verloren die Demokraten im Repräsentantenhaus zehn Sitze und konnten im Senat nicht einen einzigen hinzugewinnen. Allerdings zeigt eine genauere Untersuchung der Wahlkreisergebnisse auch, dass einige Demokraten in ihren Wahlkreisen besser als ihr Präsident abgeschnitten haben. ${ }^{7}$ Die Wirkungen Obamas auf den Wahlausgang sind also ambivalent zu bewerten.

Am 4. November 2008 konnten die Demokraten in beiden Kammern des Kongresses ihre Mehrheiten ausbauen, was die Parlamentspräsidentin Nancy Pelosi mit den Worten „wirklich historisch" ${ }^{8}$ bezeichnete. Im Repräsentantenhaus vergrößerte sich die Mehrheit von 236 auf 257 Sitze, deutlich über der erforderlichen Zahl von 218. Damit liegen die Demokraten nun auf etwa demselben Niveau wie vor der Wahl 1994, bei der sie ihre 40 Jahre währende Mehrheit einbüßten. Insgesamt konnten sie die Zahl ihrer Abgeordne-

Tedisco durchsetzen; vgl. David M. Halbfinger, Democrat Is Winner of a New York House Race, in: The New York Times vom 24. April 2009.

4 Vgl. Susan Page, Disapproval of Bush Breaks Record, in: USA Today vom 21. April 2008, vgl. auch die Übersicht für das Jahr 2008 unter http://www.pollingreport.com/BushJob.htm (Abruf am 30. März 2009).

5 Vgl. Dan Eggen, On a Farewell Tour of Sorts, Bush Reflects On His Record, in: The Washington Post vom 6. Dezember 2008, und Kate Zernike, Bush's Legacy vs. the 2008 Election, in: The New York Times vom 14. Januar 2007.

6 Vgl. David Baumann, Hard Times Put Hill to Work, in: CQ Weekly vom 7. Dezember 2008.

7 Vgl. Carl Hulse, Not All New Democrats Rode an Obama Tide, in: The New York Times vom 7. Dezember 2008.

8 Vgl. Perry Bacon Jr., Democrats Win 18 More House Seats, in: The Washington Post vom 6. November 2008. 
ten innerhalb der letzten beiden Wahlzyklen (2006/2008) von 203 auf 257 ausbauen (vgl. Tabelle 1). Allerdings erwiesen sich Voraussagen vor der Wahl, die Demokraten könnten 30 oder mehr Sitze hinzugewinnen, als übertrieben. ${ }^{9}$ Die Republikaner verfügen im 111 . Kongress nur noch über 178 Mandate, ein Verlust von 21 Sitzen.

\section{Tabelle 1: Sitzverteilung im US-Kongress, 104. bis 111. Kongress}

\begin{tabular}{|c|c|c|c|c|c|c|c|c|}
\hline & 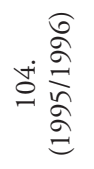 & 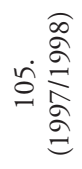 & & 용 & 离 & 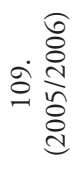 & 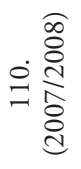 & 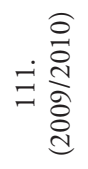 \\
\hline \multicolumn{9}{|c|}{ Repräsentantenhaus } \\
\hline $\mathrm{D}$ & 204 & 207 & 211 & 212 & 207 & 203 & 232 & 257 \\
\hline $\mathrm{R}$ & 230 & 227 & 223 & 221 & 227 & 231 & 202 & 178 \\
\hline I & 1 & 1 & 1 & 2 & 1 & 1 & 1 & 0 \\
\hline \multicolumn{9}{|c|}{ Senat } \\
\hline $\mathrm{D}$ & 47 & 45 & 45 & 50 & 48 & 44 & 49 & $58^{1}$ \\
\hline $\mathrm{R}$ & 53 & 55 & 55 & 50 & 51 & 55 & 49 & 39 \\
\hline I & 0 & 0 & 0 & 0 & 1 & 1 & 2 & 2 \\
\hline \multicolumn{9}{|c|}{$\begin{array}{l}\text { Anmerkungen: } \mathrm{D}=\text { Demokrat, } \mathrm{R}=\text { Republikaner, } \mathrm{I}=\text { Independent; an } 435 \text { (Repräsentantenhaus) feh- } \\
\text { lend: Vakanz zum Zeitpunkt der Wahl; Angaben stets zu Beginn der Legislaturperiode, spätere Verände- } \\
\text { rungen sind nicht berücksichtigt. } \\
{ }^{1} \text { Die Senatswahl in Minnesota ist noch nicht endgültig entschieden (siehe Text). } \\
\text { Quelle: Eigene Zusammenstellung. }\end{array}$} \\
\hline
\end{tabular}

Hinzu kam für die Demokraten der Vorteil, dass in den Wahlen zum Repräsentantenhaus nur etwa drei Dutzend Wahlkreise wirklich als kompetitiv angesehen werden können, das heißt die Wahrscheinlichkeit eines Umschwungs von der einen zur anderen Partei ist - abseits größerer Verwerfungen oder weit reichender, die Wahl beeinflussender Ereignisse oder Entwicklungen - relativ gering. Aufgrund des Amtsbonus der Abgeordneten und Senatoren, des Redistricting und Gerrymandering ist es für die Herausforderer von Amtsinhabern schwer, sich in der Wahl durchzusetzen.

Amtsbonus: Den Amtsinhabern bietet sich im Vergleich zu ihren Herausforderern große Vorteile. Sie sind im Wahlkreis deutlich sichtbarer und erhalten qua Amt eine finanzielle Unterstützung für die Unterhaltung von Wahlkreisbüros, für kostenlose Briefsendungen an Wähler sowie Bundesgelder für Projekte im Wahlkreis.

Redistricting: In den USA wird alle zehn Jahre ein Zensus durchgeführt, auf dessen Basis die Kongresswahlbezirke im Bedarfsfall so zugeschnitten werden, dass in jedem Wahlkreis etwa gleich viele Bürger leben. So haben nach dem letzten Census im Jahre 2000 die großen Staaten im Nordosten (zum Beispiel Illinois, Michigan, New York, Pennsylvania) Kongressmandate (und damit - nicht zu vergessen - auch Wahlmännerstimmen in der Präsidentschaftswahl) eingebüßt, mehrere Staaten im Süden und Südwesten der USA (zum

9 Vgl. ebenda; durch Neuauszählungen bei knappen Wahlkreisergebnissen ist diese Zahl inzwischen auf 21 angestiegen. 
Beispiel Arizona, Florida, Texas) haben Mandate hinzugewonnen. Dieser Trend dürfte sich aller Voraussicht nach auch beim nächsten Census im Jahre 2010 fortsetzen. ${ }^{10}$

Gerrymandering: Demokraten wie Republikaner versuchen gleichermaßen, den Neuzuschnitt der Wahlkreise zum eigenen Vorteil zu beeinflussen. Namensgeber dieses Phänomens ist Gouverneur Elbridge Gerry (Massachusetts), der 1812 ein Gesetz zur Neueinteilung von Wahlkreisen unterschrieb, das seine (Republikanische) Partei extrem begünstigte. ${ }^{11}$ Inzwischen gibt es Computerprogramme, die vorhandene Wahlkreise automatisch so schneiden können, dass die eine oder andere Partei maximal profitiert. Diese Entwicklung hat dazu geführt, dass im allergrößten Teil der Wahlkreise bereits vor der Wahl der spätere Sieger so gut wie feststeht. Die Wiederwahlquote der Amtsinhaber liegt daher seit vielen Jahren bei 95 bis 99 Prozent, im Senat etwas niedriger. Interessant für die Parteien sind demzufolge insbesondere so genannte Open Seats - Wahlkreise, in denen der Amtsinhaber nicht wieder antritt.

In der Kongresswahl von 2008 gab es gleich eine ganze Reihe solcher Sitze. So traten insgesamt 27 Mitglieder der Republikaner im Repräsentantenhaus nicht zur Wiederwahl an, auf Seite der Demokraten waren es nur sechs, die sich nicht um ein neues Mandat bewarben. Vier Amtsinhaber verloren den Kampf um die Nominierung für ihre Partei im Vorwahlprozess (drei Republikaner, ein Demokrat), 19 weitere verloren in der Hauptwahl gegen ihre jeweiligen Gegenkandidaten (14 Republikaner, fünf Demokraten). Dennoch sind nur 54 Neulinge in das Repräsentantenhaus eingezogen, weit entfernt von der Höchstzahl von 118 im Jahre 1949 und ebenfalls weit entfernt vom seit 1913 gemessenen Durchschnitt von 82 Neulingen. ${ }^{12}$ Auch dies deutet - neben anderen Ursachen - darauf hin, dass es bei Kongresswahlen immer weniger kompetitive Wahlkreise gibt. ${ }^{13}$

In den Wahlen von 2004, 2006 und nun auch 2008 setzt sich damit eine Tendenz fort, die bereits seit geraumer Zeit zu beobachten ist. 2000 gab es noch 50 Wahlkreise mit echter Konkurrenz, 1992 waren es sogar noch 100. Aufgrund der zunehmenden innerparteilichen Homogenität in den Wahlkreisen findet die wirkliche Auswahl des künftigen Mandatsinhabers heute in den Vorwahlen der dominierenden Partei statt, nicht mehr in der Hauptwahl. Dies führt dazu, dass die nominierten Demokraten deutlich nach links und ihre Republikanischen Kontrahenten deutlich nach rechts tendieren, da sie sich in der Vorwahl an der jeweiligen Parteibasis durchsetzen müssen. Ist die Vorwahl gewonnen, können die Kandidaten der überlegenen Partei relativ sicher sein, auch die Hauptwahl für sich entscheiden zu können. Konsequenz dieser Entwicklung ist also, dass sich das Parteiensystem aus Demo-

10 Vgl. Juliet Eilperin, Fight Club Politics. How Partisanship is Poisoning the House of Representatives, Lanham 2006, S. 89 - 113; vgl. auch Richard E. Cohen, Up to 13 Seats Could Be Reapportioned in 2010, in: National Journal Online vom 22. Dezember 2008, http://www.nationaljournal.com/njonline/no_20081222_1323.php (Abruf am 5. Januar 2009), und ders., Redistricting Does GOP No Favors, in: National Journal Online vom 20. November 2008, http://www. nationaljournal.com/election2008/el_20081120_7633.php (Abruf am 25. November 2008).

11 Vgl. Birgit Oldopp, Das politische System der USA. Eine Einführung, Opladen 2005, S. 204 f.

12 Vgl. Kate Phillips, New Voices in Congress Will Change the Tone of the Democratic Majority, in: The New York Times vom 7. Januar 2009.

13 Vgl. zu den Ursachen und den Folgen dieser Entwicklung Bruce I. Oppenheimer, Deep Red and Blue Congressional Districts: The Causes and Consequences of Declining Party Competitiveness, in: Lawrence C. Dodd / Bruce I. Oppenheimer (Hrsg.), a.a.O. (Fn. 2), S. 135 - 157. 
kraten und Republikanern weiter polarisiert und es im Endeffekt wichtiger ist, die eigenen politischen Ziele wenn nicht im Parlament durchzusetzen, so doch zumindest öffentlich zu thematisieren, als dass ernstgemeinte Anstrengungen unternommen werden, im parlamentarischen Aushandlungsprozess Kompromissfähigkeit und Verhandlungsbereitschaft zu zeigen, um zu möglichst optimalen Ergebnissen zu kommen. ${ }^{14}$

Im Repräsentantenhaus konnten die Demokraten 2008 den Verlust von vier Abgeordneten kompensieren, darunter die Niederlage des in einen Sexskandal verwickelten Tim Mahoney (Florida) und von Nick Lampson, der zwei Jahre zuvor in Texas überraschend gegen den langjährigen ehemaligen Mehrheitsführer der Republikaner, Tom DeLay, gewonnen hatte. Mehr als ein Dutzend Republikanischer Amtsinhaber wurde geschlagen, darunter der letzte Republikanische Kongressabgeordnete in den Staaten Neuenglands, Christopher Shays (Connecticut), der seinen Distrikt seit 1987 in Washington repräsentiert hatte. ${ }^{15}$

Im Senat konnten die Demokraten ihre Mandatszahl von 49 auf zunächst 57 ausbauen, scheiterten jedoch bei dem Versuch, eine - zeitweilig erreichbar scheinende - Mehrheit von 60 Sitzen zu erzielen. Die Republikaner verloren 2008 neun Mandate und kamen vorerst auf 40 Sitze. Die beiden Senatoren Joseph I. Lieberman (Connecticut) und der Sozialist Bernie Sanders (Vermont) gelten als Unabhängige (Independents), stimmen in der Regel jedoch mit der Demokratischen Fraktion. Mit dem überraschenden Übertritt von Senator Arlen Specter (Pennsylvania) von den Republikanern zu den Demokraten am 28. April 2009 konnten die Demokraten einen weiteren Sitz im Senat hinzugewinnen. ${ }^{16}$

In den Senatswahlkämpfen des Jahres 2008 kam es zu einer ganzen Reihe von interessanten Duellen, bei denen die Demokraten in gleich mehreren umkämpften Bundesstaaten den Sieg davontrugen.

Alaska: Erst in der zweiten Novemberhälfte 2008 entschied sich die Wahl in Alaska, als der Republikanische Amtsinhaber und langjährige Senator Ted Stevens (der Alaska seit 1968 im Senat vertreten hatte) Mitte November 2008 seine Wahlniederlage gegen den demokratischen Kandidaten und Bürgermeister von Anchorage, Mark Begich, einräumte. ${ }^{17}$ Hatte Stevens zunächst noch in Führung gelegen, so reduzierte sich der Vorsprung im Rahmen der sich hinziehenden Auszählung immer weiter, bis Mitte November Begich die Führung übernahm, zunächst mit drei, dann mit 800 Stimmen. Am Ende betrug der Vorsprung des Demokraten laut amtlichem Endergebnis 3724 Stimmen (bei 315000 abgegebenen Stim-

14 Vgl. näher zur hier beschriebenen Polarisierung der politischen Parteien Patrick Horst, Der neue Republikanische Kongress: Polarisiert, zentralisiert und nachgiebig gegenüber dem Präsidenten, in: ZParl, 36. Jg. (2005), H. 3, S. 680 - 699, S. 681 ff.; illustriert werden diese Entwicklungen durch zwei Äußerungen von unterschiedlicher politischer Seite: „I don't object to polarization if it achieves an objective" wird dem früheren Parlamentspräsidenten Newt Gingrich zugeschrieben, und ein Mitarbeiter der heutigen Parlamentspräsidentin Nancy Pelosi äußerte: „It's not about governing. We're focused on message", beide Zitate in Juliet Eilperin, a.a.O. (Fn. 10), S. 1, S. 5.

15 Shays, der von Kritikern bereits über Jahre als RINO (Republican In Name Only) kritisiert worden war, hatte sich schon in den Wahlen zuvor jeweils nur äußerst knapp durchsetzen können, vgl. Keith B. Richburg, In Northeast, Lost in a Blue Wave, in: The Washington Post vom 11. November 2008, und Raymond Hernandez, Northeast Republicans Lose Precious Ground in Washington, in: The New York Times vom 5. November 2008.

16 Vgl. Carl Hulse / Adam Nagourney, Specter Switches Parties, More Heft for Democrats, in: The New York Times vom 28. April 2009, S. 1.

17 Vgl. Carl Hulse, Stevens Bows Out In Alaska Senate Race, in: The New York Times vom 19. November 2008. 
men). Allerdings wäre offen gewesen, ob Stevens sein Amt im Januar 2009 bei einem Wahlsieg überhaupt angetreten hätte. Am 27. Oktober 2008 wurde er wegen Korruption in sieben Anklagepunkten schuldig gesprochen. Innerhalb der Republikanischen Fraktion im Senat wurden unmittelbar nach der Wahl Stimmen laut, die Stevens einen Amtsverzicht nahelegten. ${ }^{18}$

Colorado: Mark Udall, der zuvor einen Kongresswahlbezirk von Colorado im Repräsentantenhaus in Washington vertreten hatte, konnte den Senatssitz gewinnen. Amtsinhaber Wayne Allard von den Republikanern trat nicht zur Wiederwahl an. Der Kandidat der Republikaner, Bob Schaffer, unterlag in diesem Duell früherer Kongressabgeordneter Udall deutlich mit 43 zu 53 Prozent. ${ }^{19}$

New Mexico: Zur gleichen Zeit konnte Mark Udalls Cousin, Tom Udall, der ebenfalls zuvor dem US-Repräsentantenhaus angehört hatte, den Senatssitz des nicht wieder kandidierenden Republikaners Pete Domenici erobern. Udall gewann gegen den Republikaner Steve Pearce mit 61 zu 39 Prozent. Die Udalls repräsentieren eine Familie mit langer politischer Tradition. Stewart Udall, Toms Vater, war unter den Präsidenten John F. Kennedy und Lyndon B. Johnson als Innenminister tätig; Mark Udall ist der Sohn des legendären Demokratischen Politikers Morris Udall, der in den Jahren 1961 bis 1991 den Bundesstaat Arizona im Repräsentantenhaus vertrat.

New Hampshire: Jeanne Shaheen, von 1997 bis 2003 Gouverneurin des Staates New Hampshire, konnte sich am Ende überraschend deutlich mit $52 \mathrm{zu} 45$ Prozent gegen den amtierenden Senator John Sununu, Sohn des früheren Stabschefs von Präsident George H. W. Bush im Weißen Haus, behaupten. Bereits im Jahr 2002 hatte sie gegen Sununu kandidiert, war ihm aber äußerst knapp unterlegen.

North Carolina: Hier verlor die Republikanische Senatorin Elizabeth Dole, Ehefrau des Präsidentschaftskandidaten von 1996, Robert J. Dole, deutlich mit 44 zu 53 Prozent nach nur einer Amtszeit ihren Sitz an die Demokratin Kay R. Hagan, die zuvor dem Senat von North Carolina angehört hatte.

Virginia: Einen überzeugenden Sieg mit fast zwei Dritteln der Stimmen konnte der Demokrat und frühere Gouverneur Mark R. Warner über den Republikaner Jim Gilmore erzielen, ebenfalls ein früherer Gouverneur des Staates (in Virginia verbietet eine Term Limit-Regel eine unmittelbar anschließende zweite Amtszeit). Ursprünglich als ein Präsidentschaftskandidat für 2008 gehandelt, bewarb sich Warner um den Sitz des langjährigen, nicht wieder kandidierenden Senators John W. Warner (beide sind nicht miteinander verwandt). Auch bat Warner im Sommer 2008, nicht als Vizepräsidentschaftskandidat berücksichtigt zu werden. Für Barack Obama wie für Hillary Clinton wäre er eine hervorragende Ergänzung gewesen. Beide Senatssitze Virginias werden nunmehr erstmals seit 1970 von Demokraten gehalten.

Minnesota: Das Senatsrennen zwischen Amtsinhaber Norm Coleman und dem Kabarettisten Al Franken ist auf absehbare Zeit nicht beendet. Aufgrund des knappen Wahlausgangs und der damit einhergehenden automatischen Neuauszählung (bei einem Vorsprung von weniger als 0,5 Prozent aller Stimmen) sowie darauf folgender juristischer Manöver

18 Vgl. Neil A. Lewis, Alaska Senator Stevens Is Guilty Over his Failures to Disclose Gifts, in: The New York Times vom 28. Oktober 2008.

19 Vgl. zu den einzelnen Senatswahlergebnissen: http:/www.cnn.com/ELECTION/2008/results/ full/\#S (Abruf am 1. Februar 2009). 
beider Seiten dürfte eine Entscheidung noch auf sich warten lassen. Das für die Zertifizierung der Wahl zuständige Canvassing Board hatte am 5. Januar 2009 Franken mit 225 Stimmen Vorsprung (bei 2,8 Millionen abgegebenen Stimmen) zum Sieger erklärt; die Coleman-Kampagne kündigte daraufhin an, alle zur Verfügung stehenden gerichtlichen Möglichkeiten auszuschöpfen. Anfang April 2009 begann eine weitere Auszählung bislang unberücksichtigter Stimmen, wobei sich Frankens Vorsprung von 225 auf 312 Stimmen erhöhte. ${ }^{20}$ Unterdessen hat die Führungsmannschaft der Republikanischen Fraktion im Senat angedroht, einen potenziellen Einzug Frankens in die Kammer mit Hilfe eines Filibusters zur Not zu blockieren. ${ }^{21}$

Oregon: Der Parlamentspräsident des Repräsentantenhauses von Oregon, Jeff Merkley, schaffte es bei seinem ersten bundesstaatsweiten Wahlkampf, den amtierenden Senator Gordon Smith knapp mit 49 zu 46 Prozent zu schlagen.

In einigen anderen Bundesstaaten hatten sich die Demokraten ebenfalls Hoffnungen auf elektorale Erfolge gemacht, gingen aber leer aus. ${ }^{22}$ In Georgia musste der Republikanische Senator Saxby Chambliss gegen seinen Herausforderer von den Demokraten, Jim Martin, in die Stichwahl (das Wahlrecht Georgias sieht eine solche vor, wenn kein Kandidat mehr als 50 Prozent der Stimmen erhält; Chambliss hatte im ersten Wahlgang 49,7 Prozent erhalten). Am 2. Dezember 2008 gelang es ihm schließlich, mit 57 zu 43 Prozent die Oberhand gegenüber Martin zu behalten. Damit geriet die wichtige Zahl von 60 Sitzen für die Demokraten in weite Ferne, sollte nicht doch Franken zum Senator für Minnesota ausgerufen werden.

Abgesehen von selbstgemachten Problemen der Republikaner wie etwa in Alaska zeigten die Demokratischen Senatskandidaten, dass sie in allen Teilen des Landes Wahlen gewinnen können, selbst in zum Teil traditionellen Republikaner-Hochburgen. Ob dies den besonderen Umständen der Wahl von 2008 geschuldet war oder ob die Demokraten auch mittelfristig in diesen Bundesstaaten erfolgreich sein werden, bleibt abzuwarten.

Insgesamt lautet das Fazit amerikanischer Experten, dass die Republikaner im Kongress nach dem Ausscheiden vieler politisch moderater Abgeordneter insgesamt konservativer geworden sind, die Demokratische Fraktion jedoch zur Mitte tendiert. ${ }^{23}$ Dies beruht vor allem auf dem Anwachsen der so genannten Blue Dog Democrats, einer Koalition von Abgeordneten, die programmatisch eher konservativ orientiert sind und insbesondere bei fiskalpolitisch relevanten Gesetzesvorlagen ihren Einfluss geltend machen dürften. Im Repräsentantenhaus umfasst die Blue Dog-Koalition etwa 55 bis 60 Abgeordnete, im Senat zählen dazu etwa 20 Demokraten. ${ }^{24}$ Bei den anstehenden Beratungen zu den legislativen Prioritäten der Obama-Administration dürfte diese Gruppe eine zentrale Rolle spielen.

20 Vgl. Eric Kleefeld, With Votes Counted, Franken Now Leads By 312 Votes, Talking Points Memo, 7. April 2009, http://tpmdc.talkingpointsmemo.com/2009/04/with-votes-counted-franken-nowleads-by-312-votes.php?ref=fp1 (Abruf am 8. April 2009).

21 Vgl. Aaron Blake, Cornyn Promises Filibuster on Franken, in: The Hill vom 2. Januar 2009.

22 Vgl. Carl Hulse / David M. Herszenhorn, G.O.P. Facing Tougher Battle for Congress, in: The New York Times vom 9. Oktober 2008; Alexander Bolton, Confident Dems take Senate Fight into deep-red South, in: The Hill vom 16. Oktober 2008.

23 Vgl. Norman J. Ornstein, Shifting Politics Reflect Changes in Regional Alignments, in: Roll Call, 4. November 2008, und Kate Phillips, a.a.O. (Fn. 12).

24 Vgl. Manu Raju, Centrist Democrats May Expand Their Ranks, Gain More Influence in Senate, in: The Hill vom 15. September 2008. 


\section{Die Zusammensetzung des neuen Kongresses: Sozialstruktur und Führungspositionen}

Die durchschnittliche Verweildauer der Kongressmitglieder beträgt zu Beginn des 111 . Kongresses elf Jahre für Abgeordnete (5,5 Amtszeiten) und 12,9 Jahre für Senatoren (2,2 Amtszeiten). Sie liegt damit für Abgeordnete um zwölf Monate höher als im 110. und um achtzehn Monate höher als im 109. Kongress. Am längsten war John Dingell im Repräsentantenhaus: Er trat sein Amt im Dezember 1955 an. ${ }^{25}$ Die Durchschnittszeit der Senatoren liegt sechs Monate über der des 110. und etwas mehr als zwölf Monate über der des 109. Kongresses. Senator Robert Byrd aus West Virginia ist inzwischen mit 50 Jahren im Senat der am längsten amtierende Senator aller Zeiten.

Die Zahl der weiblichen Kongressmitglieder hat mit 95 einen neuen Höchststand erreicht. Davon sind 78 Angehörige des Repräsentantenhauses (plus vier im Vergleich zum vorhergehenden Kongress) und 17 Senatorinnen (plus eins). Im Repräsentantenhaus sitzen derzeit 41 afroamerikanische Abgeordnete; nach dem Ausscheiden Obamas ist allerdings keine/r mehr im Senat vertreten. 28 Repräsentanten und drei Senatoren sind hispanischer Abstammung; neun Vertreter im Repräsentantenhaus und zwei im Senat haben asiatische Wurzeln. Im Repräsentantenhaus sitzt darüber hinaus ein indianischer Abgeordneter. Durchschnittlich sind die Repräsentanten 57 Jahre alt, die Senatoren 63,1 Jahre. ${ }^{26}$

Nach den Wahlen von 2008 ist es in beiden Kammern des Kongresses bei der Besetzung der parlamentarischen Führungspositionen zum Teil zu personellen Veränderungen gekommen. Die seit 1987 dem Repräsentantenhaus angehörende Nancy Pelosi wurde erneut als Parlamentspräsidentin (Speaker) nominiert und am 3. Januar 2009 für eine zweite Amtszeit gewählt. Mehrheitsführer (Majority Leader) bleibt Steny Hoyer aus Maryland, Majority Whip bleibt James E. Clyburn aus South Carolina. Neu besetzt werden musste der Posten des Democratic Caucus Chairman, der nominell vierthöchsten Position, die die Demokraten im Repräsentantenhaus zu vergeben haben, nachdem Rahm Emanuel aus Illinois zum Stabschef des Weißen Hauses durch Obama berufen worden war. An seine Stelle trat John B. Larson aus Connecticut, der von seinem Posten als Vizevorsitzender der Demokratischen Fraktion aufrückte; Larsons alte Position übernahm Xavier Becerra aus Kalifornien. Den Vorsitz des Democratic Congressional Campaign Committee (DCCC), des für die Unterstützung und Wiederwahl der Demokratischen Abgeordneten zuständigen Gremiums, hat seit der Wahl 2006 Chris van Hollen aus Maryland inne, der zugleich den außerordentlichen Posten des Assistenten der Parlamentspräsidentin bekleidet.

Auf Republikanischer Seite wurde John Boehner (Ohio) erneut zum Minority Leader gewählt. Minority Whip wurde Eric Cantor (Virginia). Mike Pence aus Indiana hat als Republican Conference Chairman den dritthöchsten Führungsposten der Republikaner inne. Thaddeus McCotter (Michigan) ist der House Republican Policy Committee Chairman.

25 Vgl. Perry Bacon Jr., Dingell is Longest-Serving Member, in: The Washington Post vom 11. Februar 2009.

26 Vgl. für diese und weitere Daten: Mildred Amer / Jennifer E. Manning, Membership of the 111th Congress: A Profile, CRS Report for Congress R40086, 31. Dezember 2008, S. 5 f.; vgl. auch: http://www.cqpolitics.com/cq-assets/cqmultimedia/pdfs/NM-guide-statistics.pdf (Abruf am 6. April 2009), und Carl Hulse, Change in Congress More Than a Slogan, in: The New York Times vom 21. November 2008. 
Im Senat wurde Joseph Biden (Delaware) mit der Übernahme des Amtes des Vizepräsidenten der USA automatisch Präsident des Senats. In seiner Abwesenheit fungiert Senator Robert Byrd (West Virginia) als President Pro Tempore. Auf Demokratischer Seite füllt der Senator Harry Reid (Nevada) wie schon im 110. Kongress das Amt des Majority Leaders aus. Zur Seite steht ihm als Majority Whip Richard Durbin (Illinois). Der New Yorker Senator Charles Schumer ist Democratic Caucus Vice-Chairman Secretary. Der Junior-Senator aus New Jersey, Robert Menendez, leitet für die nächsten beiden Jahre das Democratic Senatorial Campaign Committee (DSCC), die Wahlkampforganisation der Senatsdemokraten.

Unklar war nach der Wahl vom November 2008 zunächst, wie die Demokraten im Senat in der Causa Joe Lieberman verfahren würden. Der inzwischen als Unabhängiger geltende Lieberman, im Jahr 2000 noch Vizepräsidentschaftskandidat des damaligen Demokratischen Spitzenkandidaten Al Gore, hatte zuvor durch demonstrative Unterstützung von Präsident Bush in der Irakkriegsfrage sowie durch seinen Auftritt auf dem Wahlparteitag der Republikanischen Partei im August 2008, auf dem er sich wiederholt kritisch über Obama äußerte und seine Unterstützung für John McCain herausstrich, für Irritationen im Demokratischen Lager gesorgt. Für kurze Zeit wurde Lieberman sogar als Vizepräsidentschaftskandidat von $M c$ Cain gehandelt, bevor sich dieser vor allem mit Blick auf die konservative Basis der Partei für die Gouverneurin von Alaska, Sarah Palin, entschied. Schon 2006 hatte Lieberman die Demokratische Vorwahl in seinem Heimatstaat Connecticut verloren, trat zur Hauptwahl dann als Unabhängiger an, gewann und konnte so in den Senat zurückkehren. Im Senat schloss sich Lieberman jedoch wieder der Demokratischen Fraktion an und sorgte für die 51. Stimme, die den Demokraten die Mehrheit im Senat brachte. Im Gegenzug erhielt er den Vorsitz des prestigeträchtigen Ausschusses für Heimatschutz. Nach der Wahl von 2008, als die Mehrheitsverhältnisse im Senat deutlicher hervortraten, drängten mehrere Demokratische Senatoren darauf, Lieberman aus der Fraktion auszuschließen beziehungsweise ihm den Ausschussvorsitz zu nehmen. Mit einer Mehrheit von 42 zu 13 Stimmen verzichtete die Fraktion Mitte November 2008 jedoch darauf und entzog Lieberman „nur“ seinen Vorsitz im Unterausschuss - nicht zuletzt deshalb, weil zu jenem Zeitpunkt eine Mehrheit von 60 Sitzen für die Demokraten zwar unwahrscheinlich, aber nicht gänzlich ausgeschlossen war. ${ }^{27}$

Auf Republikanischer Seite bleibt Mitch McConnell (Kentucky) auch im 111. Kongress der Minority Leader und Jon Kyl aus Arizona Minority Whip. Lamar Alexander (Tennessee) bekleidet das Amt des Republican Conference Chairman. John Cornyn (Texas) leitet das National Republican Senatorial Committee (NRSC), das sich um die Wiederwahl der Republikanischen Senatoren sowie um die Rekrutierung geeigneter Herausforderer bemüht.

\section{Die entscheidende Zahl im Senat: 60 Sitze}

Trotz ihrer Zugewinne haben die Demokraten die entscheidende Zahl der Sitze im Senat nicht erreicht: Mit 60 Stimmen nämlich kann ein so genannter Filibuster verhindert werden. ${ }^{28}$ Als Filibuster wird eine Marathonrede bezeichnet, mit der eine Minderheit (genauer:

27 Vgl. Paul Kane, Democrats Discuss Lieberman's Future; Obama Wants to Keep Him in Caucus, in: The Washington Post vom 12. November 2008.

28 Vgl. zum Filibuster Walter J. Oleszek, Congressional Procedures and the Policy Process, Washington 2001 , S. $228-236$. 
ein einzelner Senator) die Verabschiedung einer Gesetzesvorlage durch die Mehrheitsfraktion zu verhindern oder wenigstens zu verschieben versucht, wobei hinter den Kulissen zumeist fieberhaft darauf hingearbeitet wird, einzelne Senatoren der Mehrheitsfraktion von dem Beschluss abzubringen beziehungsweise einen von allen tragbaren Kompromiss herbeizuführen. Möglich wird diese Taktik durch eine im Vergleich zum Repräsentantenhaus sehr liberale Geschäftsordnung, derzufolge die grundsätzliche Redezeit der einzelnen Senatoren nicht begrenzt ist. Heutzutage kann ein Filibuster mit 60 Stimmen beendet werden - natürlich unter der Voraussetzung, dass sich alle Mitglieder einer Fraktion (in der Regel der Mehrheitsfraktion) einig sind, was im Senat nicht selbstverständlich ist. Da es im Kongress als Parlament eines präsidentiellen Regierungssystems in sachpolitischen Fragen keine direkte Fraktionsdisziplin gibt (in personalpolitischen Fragen ist dies in der Regel anders), fühlt sich jeder Senator vorrangig zunächst seinem eigenen Bundesstaat verpflichtet (der Abgeordnete im Repräsentantenhaus demgemäß zunächst seinem Wahlkreis), bevor er politische Rücksicht auf seine Partei beziehungsweise den aus derselben Partei stammenden Präsidenten walten lässt.

Sollte es nicht gelingen, Franken als 60. Demokratischen Senator ins Amt zu bringen, wäre der Demokratische Mehrheitsführer Reid vermutlich darauf angewiesen, einen breit angelegten Politikansatz zu verfolgen. Er dürfte versuchen, moderate Republikaner wie Olympia J. Snow und Susan M. Collins (beide Maine) für einzelne Projekte zu gewinnen und den Republikanern erst gar keine Veranlassung zu geben, auf den Filibuster zurückzugreifen. Bei der endgültigen Verabschiedung des Stimulus-Pakets am 12. Februar 2009 waren es Snow, Collins und der damals noch für die Republikanische Partei stimmende Specter, die als einzige Mitglieder ihrer Fraktion im Senat mit Ja stimmten; im Repräsentantenhaus war das Ergebnis ein striktes „Party Vote“, das heißt, die Demokraten stimmten mehrheitlich für, die Republikaner gegen die Vorlage. ${ }^{29}$

Ein anderes Abstimmungsverhalten zeigte sich dagegen in beiden Kammern bereits wenige Wochen später bei der Verabschiedung von Obamas erstem Haushaltsplan im März 2009..$^{30}$ Im Senat wurde der Haushaltsplan mit 55 zu 43 und damit ohne Stimmen der Republikanischen Seite angenommen. Auch im Repräsentantenhaus gab es bei einem Abstimmungsergebnis von 233 zu 196 ein eindeutiges Party Vote. ${ }^{31}$ Dabei hatte Obama auch zu unkonventionellen Methoden, einschließlich eines öffentlichkeitswirksamen Besuchs im Kongress, gegriffen, um auf Capitol Hill einen überparteilichen Konsens zu erzielen. ${ }^{32} \mathrm{Um}$ fragen zeigen, wie die breite Öffentlichkeit die überparteiliche Zusammenarbeit bewertet:

29 Vgl. Sheryl Gay Stolberg / Adam Nagourney, Partisan Fight Endures as Stimulus Bill Signed, in: The New York Times vom 17. Februar 2009; Peter Baker, Bipartisanship Isn't So Easy, Obama Sees, in: The New York Times vom 13. Februar 2009; Jackie Calmes, House Passes Stimulus Plan With No G.O.P. Votes, in: The New York Times vom 29. Januar 2009; vgl. zur Motivation Specters dessen Erläuterung seiner Motive in: Why I Support the Stimulus, in: The Washington Post vom 9. Februar 2009; vgl. zum Begriff des Party Vote Emil Hübner, Das politische System der USA. Eine Einführung, München 2001, S. $111 \mathrm{f}$.

30 Vgl. Carl Hulse, Budgets Approved, With No G.O.P. Votes, in: The New York Times vom 3. April 2009; vgl. auch Andrew Taylor, Congress Backs Some Obama Goals, But Not All, in: The Washington Post vom 5. April 2009.

31 Vgl. House, Senate Approve Obama Budget, in: The Los Angeles Times vom 3. April 2009.

32 Vgl. Andrew Taylor, a.a.O. (Fn. 30), und Jackie Calmes, Partisanship Is a Worthy Foe in Debate on Stimulus, in: The New York Times vom 7. Februar 2009, und Carl Hulse, Short-Circuiting Bipartisanship Is Nothing New for Congress, in: The New York Times vom 14. Februar 2009. 
Zu Beginn des Jahres 2009 waren 50 Prozent der Befragten ungewöhnlich optimistisch, dass die beiden politischen Lager zusammenarbeiten würden, 39 Prozent befürchteten vermehrt Auseinandersetzungen zwischen den Parteien. Im April 2009 haben sich die Werte deutlich verändert: Nun sahen 53 Prozent verstärkte parteipolitische Auseinandersetzungen (plus 14 Prozent), nur 25 Prozent glaubten noch an mehr Kooperation (minus 25 Prozent). Immerhin 22 Prozent schätzten die Lage unverändert ein oder haben keine Meinung (plus elf Prozent). ${ }^{33}$

\section{Der Kongress vor der Wahl 2008: „A Broken Branch“?}

Kongressbeobachter wie Thomas E. Mann und Norman J. Ornstein diagnostizieren angesichts der gesetzgeberischen Leistungen des Kongresses in den letzten Jahren eine „Broken Branch“ ${ }^{34}$, die unzureichende Erfüllung der von der Verfassung vorgegebenen Aufgaben: $\ldots$... to engage in responsible and deliberative lawmaking, police the ethical behavior of its members, and check and balance the other branches ... [This decline] included a loss of institutional patriotism among its members, an abdication of constitutional responsibility vis-à-vis the executive, the demise of regular order (in committee, on the floor and in conference), and the consequent deterioration of the deliberative process - the signature comparative advantage of Congress as a legislative body. “ 35

Natürlich ist die Arbeit des 110. Kongresses von tiefen Gräben zwischen den Parteien beeinflusst gewesen, von knappen Mehrheiten, vom wiederholten Gebrauch beziehungsweise der Androhung eines Filibusters sowie der Anwesenheit eines Republikanischen Präsidenten im Weißen Haus. Die Möglichkeiten einer Demokratischen Mehrheit im Kongress schienen also begrenzt, trotzdem weist die Nutzung parlamentarischer Instrumente in dieser Zeit einige aufschlussreiche Besonderheiten auf.

Ins Auge fällt vor allem der sprunghafte Anstieg und die Themenbreite der Anhörungen, insbesondere im Repräsentantenhaus (vgl. Tabelle 2). Diese Zahlen übersteigen nicht nur die Anhörungen im vorherigen, von den Republikanern dominierten 109. Kongress deutlich, sondern auch jene im 104. Kongress, als die Republikaner erstmals nach vierzig Jahren die Mehrheit in beiden Häusern übernommen hatten und mit Bill Clinton einem Präsidenten der Demokratischen Partei gegenüberstanden.

Ein großer Teil der Anhörungen im 110. Kongress war dem Thema Irak gewidmet, das unter der Republikanischen Mehrheit als Gegenstand parlamentarischer Kontrolle weitgehend vernachlässigt worden war. Die Rolle des Justizministeriums bei der Entlassung mehrerer Staatsanwälte, die Situation der Irak- und Afghanistan-Kriegsveteranen, die Vergabe von staatlichen Aufgaben an Unternehmen wie Blackwater und Halliburton - all diese

33 Vgl. die detaillierten Zahlen in Pew Research Center: Partisan Bickering is Back, Public Says, 8. April 2009, http://pewresearch.org/pubs/1184/partisanship-back-obama-economic-confidence-high-gop-image-low (Abruf am 9. April 2009).

34 Zuerst hier: Thomas E. Mann / Norman J. Ornstein, The Broken Branch. How Congress is Failing America and How to Get It Back on Track, New York 2006, S. $14-46$ und S. $141-191$.

35 Sarah A. Binder / Thomas E. Mann / Norman J. Ornstein / Molly Reynolds, Mending the Broken Branch: Assessing the 110th Congress, Anticipating the 111th, Januar 2009, online verfügbar unter http://www.brookings.edu/_/media/Files/rc/papers/2009/0108_broken_branch_binder_ mann/0108_broken_branch_binder_mann.pdf (Abruf am 6. April 2009), S. 2. 
Themen waren Gegenstand von Ermittlungen im Kongress. In der zweiten Session (2008) kamen (wenn auch hauptsächlich als ex post-Untersuchung) die Hypotheken-, Finanz- und Wirtschaftskrise hinzu. Auch in Bezug auf die Zuständigkeitsbereiche einzelner Ministerien und Behörden wurden die Ausschüsse des Kongresses aktiv. ${ }^{36}$

Es hat den Anschein, dass der 110. Kongress Handlungs- (und hier vor allem Kontroll-) Möglichkeiten gegenüber der Exekutive zurückgewonnen hat, die in den Jahren zuvor, teils selbstverschuldet, aufgegeben worden waren. Die Bush-Administration begründete ihren Primat mit zweierlei Argumenten: Zum einen wurde auf die einheitliche Exekutive (Unitary Executive) verwiesen, zum anderen wurde die Überzeugung vertreten, in Zeiten eines zeitlich nicht befristeten Krieges (gegen den Terror) komme der Exekutive ohnehin eine Vorrangstellung zu. Die Bilanz der Konsequenzen für den Kongress fällt eindeutig aus: "Congress actively discouraged oversight of the executive branch; held tightly constrained and limited hearings when abuses by the executive, such as Abu Ghraib, occurred or were alleged; deferred almost totally to the president on issues like surveillance at home and abroad; failed to challenge the president's assertions of constitutional authority to ignore provisions of laws via signing statements; and did not push for testimony or documents from the White House when issues, scandals or problems arose. “37 Im Vergleich zu den Jahren unter „Unified Government“ fällt das Fazit für den 110. Kongress im Rahmen eines „Divided Government" zwar gemischt, aber doch eher positiv als negativ aus. ${ }^{38}$ Der 111 . Kongress hat nun, wiederum in einem Unified Government, die Chance, an dieses Wiedererwachen der eigenen Bedeutung anzuknüpfen und den Trend zu festigen.

\begin{tabular}{|c|c|c|c|}
\hline \multicolumn{4}{|l|}{ Repräsentantenhaus } \\
\hline & $\begin{array}{c}\text { 104. Kongress } \\
(1995 / 1996)\end{array}$ & $\begin{array}{c}\text { 109. Kongress } \\
(2005 / 2006)\end{array}$ & $\begin{array}{c}\text { 110. Kongress } \\
(2007 / 2008)\end{array}$ \\
\hline \multicolumn{4}{|l|}{ Anhörungen } \\
\hline Ständige Ausschüsse und Unterausschüsse & 901 & 960 & 1403 \\
\hline Bewilligungsangelegenheiten & 440 & 242 & 362 \\
\hline Irak & 0 & 84 & 193 \\
\hline \multicolumn{4}{|l|}{ Senat } \\
\hline Ständige Ausschüsse und Unterausschüsse & 509 & 595 & 742 \\
\hline Bewilligungsangelegenheiten & 206 & 154 & 146 \\
\hline Irak & 0 & 77 & 93 \\
\hline \multicolumn{4}{|c|}{$\begin{array}{l}\text { Anmerkungen: 104. Kongress: Republikaner erstmals seit } 40 \text { Jahren in der Mehrheit, 109. Kongress: } \\
\text { Republikaner behaupten die Mehrheit, 110. Kongress: Demokraten übernehmen die Mehrheit. } \\
\text { Quelle: nach Sarah A. Binder / Thomas E. Mann / Norman J. Ornstein / Molly Reynolds, a.a.O. (Fn. 35), } \\
\text { S. } 39 \text { f. }\end{array}$} \\
\hline
\end{tabular}

Mann und Ornstein stützen ihre Befunde einer „Broken Branch“ aber nicht nur auf das Verhältnis des Kongresses zur Exekutive, sondern auch auf die Art und Weise, wie in den beiden Kammern der parlamentarische Prozess abläuft und politische Entscheidungen fal- 
len. Im Repräsentantenhaus werden die Abläufe von einer rigiden, die Mehrheitsfraktion stark bevorteilenden Geschäftsordnung bestimmt. ${ }^{39}$ Mit Hilfe von so genannten Open oder Closed Rules kann der parlamentarische Entscheidungsprozess von Seiten der Mehrheitsfraktion gesteuert werden. ${ }^{40}$ In den letzten Jahren hat sich herauskristallisiert, dass die Mehrheitsfraktion beziehungsweise deren Führung mit dem immer häufigeren Rückgriff auf Closed Rules mehr und mehr den gewöhnlichen Politikprozess umgeht. Mit dem Ziel besserer Steuerung wird auf ausführliche, überparteiliche Beratungen in den Ausschüssen und im Plenum verzichtet sowie die strikte Konzentration auf die eigene politische Seite gepflegt. Bei den Open Rules ist dagegen eine eingehende Debatte sowie die umfassende Möglichkeit zu Änderungsanträgen gegeben. Tabelle 3 zeigt, dass die Zahl der Open Rules in den letzten Jahren stark zurückgegangen ist, die Closed Rules dagegen sehr stark zugenommen haben. Im Falle einer so genannten Suspension werden die geltenden Regeln für eine Zeitlang aufgehoben, um direkt zu Abstimmungen beziehungsweise Entscheidungen zu kommen. Auch diese Zahl ist erheblich gewachsen. Schließlich sieht die Geschäftsordnung eine Motion to Recommit vor, also die Rücküberweisung einer Vorlage vom Plenum in die Ausschüsse zur erneuten Beratung. Dies ist für die Minderheit im Kongress häufig die letzte Möglichkeit, die endgültige Abstimmung zu verhindern; sie hofft darauf, dass die Vorlage im erneuten Ausschussprozess hängen bleibt (oder in veränderter Form ins Plenum zurückkehrt). Diesbezügliche Anträge waren im 110. Kongress nicht selten, allerdings waren nur 24 Anträge von Erfolg gekrönt.

\begin{tabular}{|l|c|c|c|}
\hline Tabelle 3: Nutzung von parlamentarischen Instrumenten im Repräsentantenhaus \\
\hline & $\begin{array}{c}104 . \text { Kongress } \\
(1995 / 1996)\end{array}$ & $\begin{array}{c}109 . \text { Kongress } \\
(2005 / 2006)\end{array}$ & $\begin{array}{c}110 . \text { Kongress } \\
(2007 / 2008)\end{array}$ \\
\hline Open Rules & 69 & 22 & 12 \\
\hline Closed Rules & 25 & 54 & 87 \\
\hline Suspensions & 390 & 922 & 1616 \\
\hline Motions to Recommit insgesamt & 68 & 57 & 122 \\
\hline Davon erfolgreich & 4 & 0 & 24 \\
\hline $\begin{array}{l}\text { Quelle: nach Sarah A. Binder / Thomas E. Mann / Norman J. Ornstein / Molly Reynolds, a.a.O. (Fn. 35), } \\
\text { S. 39. }\end{array}$
\end{tabular}

Im Senat verläuft der parlamentarische Prozess weniger strikt, stärker deliberativ und problemlos, solange der Mehrheitsführer die (unausgesprochene) Unterstützung seiner Kollegen hat. Dies heißt aber auch, dass bereits ein einzelner Senator die Abläufe verzögern

39 So kann der Parlamentspräsident mit Hilfe des Rules Committee die prozeduralen Regeln festlegen, die für die Debatte wichtiger Gesetze maßgeblich sind (und damit den Ausgang der Debatte und Abstimmung weitgehend determinieren). Weiterhin übernimmt die Mehrheitsfraktion alle Ausschussvorsitze (und nicht im Verhältnis der Parteienstärke wie etwa im Bundestag) und damit die Kontrolle über die Gesetzesarbeit. Die Ratio in den Ausschüssen verschafft schließlich der Mehrheitsfraktion ein günstigeres Verhältnis zur Minderheit als im Plenum, vgl. zu den Details Walter J. Oleszek, Congress and Its Members, Washington 2000, S. 197 - 255.

40 Vgl. zu den Details Walter J. Oleszek, a.a.O. (Fn. 28), S. 148 - 180, und Eric Schickler / Kathryn Pearson, The House Leadership in an Era of Partisan Warfare, in: Lawrence C. Dodd / Bruce I. Oppenheimer (Hrsg.), a.a.O. (Fn. 2), S. 207 - 225; vgl. auch Norman J. Ornstein, Rules Changes Must Allow For More Open Process in House, in: Roll Call vom 7. Januar 2009. 
beziehungsweise im Extremfall blockieren kann. ${ }^{41}$ Die Zahl der so genannten Cloture Motions, die eine Debatte beenden und direkt zur Entscheidung kommen lassen, ist seit Mitte der neunziger Jahre stark angestiegen (vgl. Tabelle 4). Im 104. und im 110. Kongress sind relativ viele Anträge gescheitert, nur im 105. Kongress, zu Zeiten eines Unified Government unter Republikanischer Kontrolle, liegt der Wert verhältnismäßig niedrig.

\begin{tabular}{|l|c|c|c|}
\hline \multicolumn{1}{|c|}{ Tabelle 4: Nutzung von parlamentarischen Instrumenten im Senat } \\
\hline \multicolumn{1}{|c|}{ Cloture Motions } & $\begin{array}{c}104 . \text { Kongress } \\
(1995 / 1996)\end{array}$ & $\begin{array}{c}105 . \text { Kongress } \\
(2005 / 2006)\end{array}$ & $\begin{array}{c}\text { 106. Kongress } \\
(2007 / 2008)\end{array}$ \\
\hline Erfolgreich & 9 & 34 & 61 \\
\hline Gescheitert & 41 & 20 & 48 \\
\hline Zurückgezogen & 18 & 17 & 27 \\
\hline Nicht behandelt & 14 & 0 & 6 \\
\hline Quelle: Sarah A. Binder / Thomas E. Mann / Norman J. Ornstein / Molly Reynolds, a.a.O. (Fn. 35), S. 40. \\
\hline
\end{tabular}

Der Kongress kann also auch in Zeiten des Divided Government ein wichtiger Gegenspieler der Exekutive sein. Es zeigt sich aber auch, dass die Versuchung innerhalb der Mehrheitsfraktion stets groß ist, das (gewünschte) Ergebnis über den (ungeliebten) Prozess zu setzen. Bezeichnend dafür ist, dass die jeweilige Minderheitsfraktion diese Entwicklungen mit deutlicher Kritik versieht; ist man jedoch selbst in der Position der Mehrheit, werden die kritisierten Verfahren fortgeführt. ${ }^{42}$

\section{Perspektiven des Unified Government unter Präsident Obama}

Die zwei Jahre bis zur Zwischenwahl im November 2010 werden zeigen, wie tragfähig die neue vergrößerte Demokratische Mehrheit im Kongress ist. Sie werden auch zeigen, ob sich die im 110. Kongress zu beobachtende Tendenz zur Rückkehr der institutionellen Machtbalance zwischen Exekutive und Legislative fortsetzt und der Kongress vermehrt die Regierung kontrolliert und deren Gewicht austarieren kann.

Im präsidentiellen Regierungssystem der USA stehen sich laut Verfassung Exekutive und Legislative, Weißes Haus und Kongress, in einem System der Checks and Balances diametral gegenüber - anders als etwa im parlamentarischen System der Bundesrepublik Deutschland, in dem die Regierung aus dem Parlament hervorgeht und kontinuierlich von einer parlamentarischen Mehrheit getragen wird. Obama kann sich demnach nicht darauf verlassen, dass er für seine Gesetzgebungsvorhaben automatisch eine parlamentarische Mehrheit bekommt, auch nicht bei seiner eigenen Partei, sondern er muss für jede Vorlage eine neue temporäre parlamentarische Koalition zusammenstellen.

Unmittelbar nach der Wahl 2008 kündigte die Präsidentin des Repräsentantenhauses Pelosi an, dass die Demokraten „aus der Mitte heraus“43 regieren würden. Für Präsident

41 Vgl. zum Prozess im Senat Walter J. Oleszek, a.a.O. (Fn. 28), S. 207 - 244, und C. Lawrence Evans / Daniel Lipinski, Obstruction and Leadership in the U.S. Senate, in: Lawrence C. Dodd I Bruce I. Oppenheimer (Hrsg.), a.a.O. (Fn. 2), S. $227-248$.

42 Vgl. Sarah A. Binder/Thomas E. Mann/Norman J. Ornstein/Molly Reynolds, a.a.O. (Fn. 35), S. 8. 43 Vgl. Perry Bacon Jr., a.a.O. (Fn. 8). 
Obama wird sich bis zur nächsten Zwischenwahl die Frage stellen, ob er und seine Verbündeten im Kongress im Sinne der „Broken Branch“ auf schnelle und effiziente parlamentarische Abläufe zurückgreifen können, um zentrale Gesetzgebungsvorhaben und herausgehobene politische Projekte zu verwirklichen. Zu nennen sind in diesem Kontext insbesondere die angestrebte umfassende Reform des Gesundheitswesens, neue Ansätze in der Klimaschutzpolitik und mögliche Ausstiegsszenarien im Irak sowie eine Neuformulierung der Strategie in Afghanistan. ${ }^{44}$ Gegen den Widerstand der Republikaner (und vermutlich einiger Demokraten) dürften diese Reformen nicht umzusetzen sein. In diesem Falle müsste Obama sich auf die Größe der Demokratischen Mehrheiten im Kongress verlassen. Damit würde er sich allerdings der Kritik aussetzen, den im Wahlkampf betonten überparteilichen Konsensus aufzukündigen. Oder er - und zusammen mit ihm die Führungsmannschaft der Demokraten in den beiden Kammern des Kongresses - fühlt sich auch bei den wichtigen Projekten der Administration daran gebunden, auf die andere Seite zugehen zu wollen und ihr beim parlamentarischen Prozedere größere Mitwirkungsmöglichkeiten einzuräumen. Eher früher als später wird sich die Obama-Administration für eine dieser beiden Vorgehensweisen entscheiden müssen.

Als Vorteil kann sich erweisen, dass Obama im Vergleich zu den Republikanern im Kongress nach wie vor über außerordentlich gute Umfragewerte verfügt. Einer Umfrage zufolge haben 62 Prozent der Befragten zu Beginn des 111. Kongresses eine vorteilhafte beziehungsweise positive Einschätzung (Favorable Opinion) der Demokraten (dieser Wert liegt um fünf Prozentpunkte höher als unmittelbar vor der Wahl im Oktober 2008); nur 40 Prozent sagen dies über die Republikanische Partei. Eine solch große Diskrepanz zwischen den beiden Parteien gab es zuletzt vor gut zwanzig Jahren. Während 78 Prozent der Anhänger der Republikaner eine positive Einstellung zu ihrer eigenen Partei haben, sagen dies sehr gute 90 Prozent der Demokraten-Anhänger. Bei den Unabhängigen genießen die Demokraten mit 58 zu 38 Prozent einen 20-Punkte-Vorsprung gegenüber den Republikanern. Bemerkenswert ist zudem, dass der konstatierte vermutete Kompetenzvorsprung in nahezu allen demographischen Gruppen zu verzeichnen ist. Fragt man nach dem Kongress als Institution, so überwiegen die negativen Einstellungen mit 52 zu 40 Prozent. ${ }^{45}$ Diese Einschätzungen müssen nicht von Dauer sein beziehungsweise die Arbeit der Abgeordneten nachhaltig beeinflussen (negative Meinungen zur Arbeit des Kongresses sind über viele Jahre hinweg weitgehend unverändert geblieben). Sie geben aber den Demokraten und Obama erst einmal Handlungsspielräume bei der Umsetzung ihrer im Wahlkampf postulierten politischen Prioritäten, auch wenn das Beispiel Bush zeigt, dass temporär gutes Abschneiden in Meinungsumfragen sehr schnell ins Gegenteil umschlagen kann. Zu berücksichtigen ist zudem, dass eine Kongresswahl (wie die im Jahre 2010) Wahlkreis für Wahlkreis und Bundesstaat für Bundesstaat entschieden wird und nicht im ganzen Lande, wie etwa im Falle der Präsidentschaftswahl.

Obama und die Demokraten werden versuchen, größere Projekte in der ersten Session des 111. Kongresses, also im Laufe des Jahres 2009, zu verabschieden, denn in der zweiten

44 Vgl. Naftali BenDavid, Biggest Challenges Still Await Congress, in: The Wall Street Journal vom 4. April 2009.

45 Vgl. zu den detaillierten Zahlen: Pew Research Center: Dems' Favorability Advantage Widens. Views of Congress Negative, More Polarized, 29. Januar 2009, online verfügbar unter http://pewresearch.org/pubs/1098/democratic-favorability-advantage-widens (Abruf am 30. März 2009). 
Session im Jahr 2010 werfen die nächsten Wahlen zum Repräsentantenhaus ihre Schatten voraus; die Arbeitsatmosphäre und die Auseinandersetzungen im Kongress dürften härter und (partei-)politischer werden.

Hinsichtlich der Wahlchancen dürften die Demokraten bis auf Weiteres im Vorteil sein. Auch nach den Kongresswahlen 2010 erscheint es wahrscheinlich, dass sich Obama in beiden Häusern des Kongresses auf eine Mehrheit seiner Partei stützen kann. Dies würde ihm die Umsetzung seiner politischen Agenda erleichtern; seit Carter hat kein Präsident während der gesamten ersten Amtszeit in einem Unified Government regieren können.

Die Republikaner müssten 2010 vierzig Sitze mehr erobern, um im Repräsentantenhaus die Mehrheit zu übernehmen - ein Erfolg, der seit 1950 erst viermal erzielt wurde und die Gewinne der Demokraten in den Wahlen 2006 und 2008 übertreffen würde. Im Senat müssten die Republikaner elf Sitze hinzugewinnen; eine zweistellige Zunahme gab es seit 1950 erst zweimal. Beide Parteien werden jeweils 18 Sitze verteidigen müssen. Hinzu kommen bereits jetzt fünf offene Sitze, die die Republikaner verteidigen müssen. ${ }^{46}$ Dabei handelt es sich um die Senatoren Kit Bond (Missouri), Sam Brownback (Kansas), Judd Gregg (New Hampshire), Mel Martinez (Florida) und George Voinovich (Ohio), die 2010 nicht wieder antreten werden. Auf Demokratischer Seite hat bislang nur der Nachfolger Senator Bidens, dessen langjähriger engster Mitarbeiter Ted Kaufman, angekündigt, 2010 in einer Special Election nicht antreten zu wollen. ${ }^{47}$ Offen ist derzeit, wie sich in einer weiteren Special Election die Nachfolgerin von Hillary Clinton, Kirsten Gillibrand, schlagen wird.

Die Frage für die Republikaner wird sein, ob sie 2010 ihre derzeitige Position behaupten oder sogar verlorenen Grund wiedergewinnen können. ${ }^{48} 2008$ haben 29 Republikanische Abgeordnete mit weniger als 55 Prozent der Stimmen gewonnen. 23 davon konnten weniger Stimmen erringen als die jeweiligen Kandidaten in der Wahl von 2004. Bei 14 Kandidaten bewegte sich der Stimmenverlust im zweistelligen Prozentbereich. ${ }^{49}$ Allerdings gibt es im Repräsentantenhaus 83 Demokratische Abgeordnete, die zum Teil sehr knapp in Wahlkreisen gewonnen haben, in denen bei der Präsidentschaftswahl 2004 mit (zum Teil deutlicher) Mehrheit für Präsident Bush gestimmt worden war. Für sie wird es außerordentlich schwierig, ihren Wahlkreis 2010 zu verteidigen. Zum Vergleich: Im Repräsentantenhaus sitzen nur fünf Republikaner, die Wahlkreise repräsentieren, die 2004 von John Kerry gewonnen wurden. ${ }^{50}$

46 Vgl. Shira Toeplitz, 2010 Senate Map May Not Be Much Better for the GOP, in: Roll Call vom 6. November 2008.

47 Es wird allgemein angenommen, dass einer der Söhne Bidens sich um das Amt bewerben wird, vgl. Rita K. Farrell, A Senator Takes Office, Departure Already in Sight, in: The New York Times vom 30. Januar 2009.

48 Vgl. Charlie Cook / David Wasserman, House GOP Faces a Long March, Not a Sprint, in: National Journal Online vom 17. Dezember 2009, http://www.nationaljournal.com/njonline/ ot_20081217_3661.php (Abruf am 5. Januar 2009).

49 Richard E. Cohen / Peter Bell, Some House Republicans Remain Vulnerable, in: National Journal Election Analysis vom 19. November 2008, http://www.nationaljournal.com/election2008/ el_20081119_4434.php (Abruf am 20. November 2008).

50 Vgl. dies., Swing Districts Likely GOP Targets, in: National Journal Online vom 10. November 2008, http://www.nationaljournal.com/election2008/el_20081110_9640.php (Abruf am 2. Januar 2009). 
Traditionell schneidet die "Party in Power“ in Zwischenwahlen, wie es 2010 eine ist, schlecht(er) ab. Vom neuen Präsidenten Obama sowie den Fraktionsführungen im Kongress hängt es in den nächsten beiden Jahren wesentlich ab, wie erfolgreich die Arbeit von Exekutive und Legislative vom Wähler beurteilt wird. Von einer dauerhaften Demokratischen Mehrheit zu sprechen, wie es die Parteistrategen der Republikaner vor einigen Jahren angesichts eines Unified Government unter Präsident Bush getan haben, wäre zum gegenwärtigen Zeitpunkt verfrüht und vermutlich argumentativ nicht zu halten. ${ }^{51}$

51 Näheres dazu vgl. Hubert Silberhorn, Nachhaltige Dominanz? Präsident Bush, die Republikaner und ihr Masterplan, in: ZParl, 39. Jg. (2008), H. 1, S. 134 - 156; vgl. auch John Micklethwait I Adrian Wooldridge, The Right Nation. Conservative Power in America, S. 227 - 269, und Andy Barr, Dems talk of ,permanent progressive majority', in: Politico vom 7. November 2008, http:// www.politico.com/news/stories/1108/15407.html (Abruf am 15. Dezember 2008), sowie Robert G. Kaiser, Pollsters Debate America's Political Realignment, in: The Washington Post vom 23. November 2008; realistisch sieht der Vorsitzende des DCCC, Chris van Hollen, die Aussichten für das Wahljahr 2010: Statt einer dritten Demokratischen Welle gehe es darum, den gegenwärtigen Status Quo zu verteidigen, vgl. Andy Barr, Chris Hollen: No third Dem Wave in 2010, in: Politico vom 13. Februar 2009, http://www.politico.com/news/stories/0209/18828.html (Abruf am 7. April 2009).

\title{
Obamas Zeitenwende: Der Sieg allein ist nicht der Wechsel. Die US-Präsidentschaftswahl vom 4. November 2008
}

\author{
Bernhard Kornelius
}

Am 4. November 2008 wurde in den USA Geschichte geschrieben. Nach acht kontrovers diskutierten Jahren Bush-Administration, einer außergewöhnlich langen und stark polarisierenden Vorwahlphase sowie der teuersten Kampagne aller Zeiten vollzog sich unter den Augen der Weltöffentlichkeit und vor dem Hintergrund der weltweiten Wirtschafts- und Finanzkrise ein spektakulärer Wechsel. 44. Präsident der Vereinigten Staaten wurde mit Barack Obama zum ersten Mal ein farbiger Kandidat. Die Demokraten hatten - flankiert von Erfolgen in Senat und Repräsentantenhaus - nach zwei knappen Niederlagen in den Jahren $2000^{1}$ und $2004^{2}$ das Weiße Haus zurückerobert. Die Präsidentschaftswahl elektrisierte wie nur wenige andere Abstimmungen der letzten Jahre oder sogar Jahrzehnte: Schließlich ging es bei der Neubesetzung des weltweit wohl wichtigsten politischen Amtes um eine transnational signifikante Richtungsentscheidung.

Unabhängig von seiner politischen Tragweite und der hohen Symbolik war das Wahlergebnis per se weniger spektakulär: Der Erfolg des Demokraten Barack Obama über seinen

1 Vgl. Martin Thunert, Die US-amerikanischen Präsidentschaftswahlen vom 7. November 2000 kein Sieg Bushs, sondern eine Niederlage Gores, in: ZParl, 32. Jg. (2001), H. 3, S. 630 - 645.

2 Vgl. Michael Kolkmann, Die Präsidentschaftswahl in den USA vom 2. November 2004: Ein knapper Sieg Bushs, aber auch ein Mandat?, in: ZParl, 36. Jg. (2005), H. 2, S. 259 - 279. 